
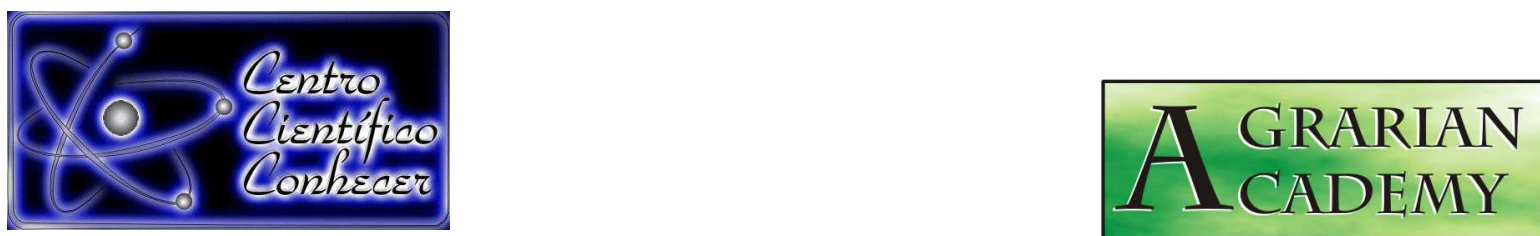

\title{
VIABILIDADE POLINICA DE Syzygium malaccense POR METODOS COLORIMETRICOS
}

\footnotetext{
${ }^{1}$ Luiz Fernando Gibbert, ${ }^{2}$ Rômulo Ribeiro, ${ }^{3}$ Sabrina Cassia Fernandes,

${ }^{4}$ Vanessa dos Santos de Mello, ${ }^{5}$ Isane Vera Karsburg

${ }^{1}$ Graduando do curso de Bacharelado em Agronomia - UNEMAT, Alta

Floresta - MT, E-mail: luizfernandogibbert@hotmail.com.

${ }^{2}$ Graduando do curso de Bacharelado em Agronomia - UNEMAT, Alta Floresta MT,

${ }^{3}$ Graduanda do curso de Bacharelado em Agronomia - UNEMAT, Alta Floresta - MT,

${ }^{4}$ Mestranda do Programa Pós Graduação em Genética e Melhoramento de Plantas UNEMAT - Alta Floresta- MT,

${ }^{5}$ Professora Adjunta da Faculdade de Ciências Biológicas e Agrárias - UNEMATAlta Floresta- MT
}

Recebido em: 15/04/2017 - Aprovado em: 22/07/2017 - Publicado em: 31/07/2017 DOI: 10.18677/Agrarian_Academy_2017a45

\begin{abstract}
RESUMO
A viabilidade do pólen é um estudo de grande importância para plantas, visto que estima-se a potencialidade reprodutora da espécie, cultivar ou população.O presente trabalho teve como objetivo avaliar a viabilidade dos grãos de pólen de Syzygium malaccense, por meio de diferentes concentrações de cloreto de 2,3,5trifeniltetrazólio (TTC) nas concentrações de 0,075\% e 0,30\% em diferentes tempos de exposição $(6,12,18$ e 24 horas) com presença e ausência de luz. Foram coletados 30 botões florais de indivíduos distintos em localidades diferentes na região de Alta Floresta-MT. As anteras dos botões florais foram retiradas e submetidas logo em seguida aos tratamentos de 2,3,5 cloreto de trifeniltetrazólio nos diferentes tratamentos e lugol $2 \%$. Ambos corantes podem ser utilizados para estimar a viabilidade, dependendo das condições para a avaliação, bem como da possibilidade de análise no momento da coleta possibilitando o uso do TTC em diferentes tempos e concentrações. Ou apenas por meio do lugol $2 \%$ em caso de não ter a possibilidade de estudo da viabilidade no momento da coleta, necessitando de processo de conservação.
\end{abstract}

PALAVRAS-CHAVE: grãos de pólen, jambo-vermelho, trifeniltetrazólio

\section{POLYNICAL VIABILITY OF SYZYGIUM MALACCENSE BY COLORIMETRIC METHODS}

\section{ABSTRACT}

The feasibility of pollen is a study of great importance for plants, since it is estimated the reproductive potential of the species, cultivar or population. The objective of this work was to evaluate the viability of the pollen grains of Syzygium AGRARIAN ACADEMY, Centro Científico Conhecer - Goiânia, v.4, n.7; p.474 2017 
malaccense, through different concentrations Of 2,3,5-triphenyltetrazolium chloride (TTC) at concentrations of $0.075 \%$ and $0.30 \%$ at different exposure times $(6,12,18$ and 24 hours) with presence and absence of light. Thirty floral buds were collected from different individuals and different locations in the Alta Floresta-MT region. The anthers of the floral buds were removed and submitted soon after the treatments of $2,3,5$ triphenyltetrazolium chloride in the different treatments and $2 \%$ lugol. Both dyes can be used to estimate the viability, depending on the conditions for the evaluation, as well as the possibility of analysis at the time of collection, allowing the use of TTC at different times and concentrations. Or only by means of lugol $2 \%$ in case of not being able to study the viability at the time of collection, necessitating conservation process.

KEYWORDS: Jambo-red, triphenyltetrazolium, pollen grains.

\section{INTRODUÇÃO}

O jambo-vermelho, Syzygium malaccense, pertence à família myrtaceae, originário da asiática, mais especificadamente da Índia e da Malásia. No Brasil, esta planta é encontrada nos estados da região Norte, Nordeste e parte do Sudeste. Esta planta pode atingir até $20 \mathrm{~m}$ de altura, popularmente conhecida por jambo. Os frutos são ricos em vitamina $A, B 1, B 12$, proteínas, antocianinas, além de cálcio, ferro e fósforo. O jambo possui altos teores de antocianinas, pelos pigmentos que estão presentes na casca dos frutos os quais dão a coloração vermelho escuro dos frutos (CAVALCANTE, 1996; KUROSAWA, 2004; MARTINS et al., 2014). As flores são hermafroditas de coloração vermelha com numerosos estames. $O$ período reprodutivo ocorre de agosto a fevereiro, quando a planta se recobre de flores, dando-lhe um aspecto bastante ornamental. $O$ desenvolvimento do fruto acontece desde a época da florada até a colheita, que ocorre de janeiro a maio. A propagação é realizada por meio de sementes (DONADIO et al., 1998).

O Syzygium malaccense pode ser consumido in natura, em formas de compota, doces, geléias, licores e aguardente, produção de corante e antioxidante natural (MARTINS et al., 2014). A planta é indicada como medicinal, as folhas são indicadas no tratamento do diabetes, mau funcionamento gastrintestinal, respiratório, bem como nas inflamações, pois possui a propriedade de inibir a ação da COX-1 durante a biossíntese das prostaglandinas, diurético e no tratamento de infecção dérmica (MELO, 2009). A produção de frutos ainda é bem reduzida e instável no Brasil (CEAGESP, em 2015). O entreposto de São Paulo comercializava em média $13.720 \mathrm{Kg}$ de frutos, sendo considerado um grande avanço dentre as frutíferas exóticas (CEAGESP, 2016).

Uma alta produtividade de frutos depende de diversos fatores entre eles presença de grande quantidade de flores com gametas viáveis. É possível analisar o potencial de reprodução de uma espécie, cultivar, ou população, por meio de estudos sobre a viabilidade polínica. Muitas espécies produzem elevado percentual de pólen viável, mas nem todos são utilizados na fertilização, por ter sido perdido pelo transporte por meio do vento ou na alimentação de insetos, desta forma o sucesso da fertilização está relacionado com a viabilidade polínica, pois quanto maior for a viabilidade maior será a probabilidade da fecundação (MARTINS et al., 2012).

Assim diferentes métodos colorímetricos são importantes a serem empregados nesses estudos, para que seja possível estimar qual método mais 
eficiente para o potencial de viabilidade polínica que possuem como pontos positivos a rapidez e o baixo custo (KUHN, 2015). Em virtude de fatores bióticos e abióticos também influenciarem na formação do grão de pólen e na interação genótipo/ambiente (local/ano), é relevante que em um programa de melhoramento genético vegetal a técnica da viabilidade polínica seja utilizada rotineiramente, a fim de agregar maior conhecimento sobre o material trabalhado, avançar na seleção e priorizar os melhores cruzamentos, excluindo ou deixando para estudos posteriores os materiais instáveis (BRAMBATTI et al., 2016). O presente trabalho teve como objetivo avaliar a viabilidade polínica de Syzygium malaccense, por meio do corante 2,3,5 cloretotrifeniltetrazólio (TTC) em duas concentrações: $0,075 \%$ e $0,30 \%$ e quatro tempos de exposição diferente em ambiente com presença e ausência de luz e lugol $2 \%$. Assim fornecendo informações sobre a viabilidade polínica que vem a auxiliar nos processos de melhoramento da espécie.

\section{MATERIAL E METODOS}

O Município de Alta Floresta, situa-se no extremo norte do Estado de Mato Grosso e a cerca de $830 \mathrm{~km}$ da capital, Cuiabá-MT, faz parte do ecossistema Amazônico. Possui uma área territorial de $9.310,27 \mathrm{~km}^{2}$ e uma população de 57.304 habitantes (ORGANIZAÇÃO PAN-AMERICANA DA SAÚDE, 2017). O clima é tropical úmido, com períodos sazonais característicos de inverno seco (maio a outubro) e verão chuvoso (novembro a abril). A temperatura pode variar entre $24^{\circ} \mathrm{C}$ e $34^{\circ} \mathrm{C} ;$ e a umidade relativa do ar, entre 15,0 e $98,0 \%$ (http://www.cptec.inpe.br(2017)).

O trabalho foi realizado no Laboratório de Citogenética e Cultura de Tecidos Vegetais da Universidade do Estado de Mato Grosso, Campus de Alta Floresta-MT. Para a realização deste trabalho foram utilizados 30 botões florais em estágio de pré antese para cada população, estas foram consideradas apresentando mais de dois indivíduos em cada local de coleta. Abaixo segue quadro com as coordenadas de coleta dos botões florais para retirada do grão de pólen:

QUADRO 1 : Identificação dos acessos, local de coleta dos botões florais em pre antese de Syzygium malaccense e dados de GPS.

\begin{tabular}{|c|c|c|}
\hline População & Local de Coleta & Coordenadas \\
\hline Pop.1 & Alta Floresta - MT & Sul: $9{ }^{\circ} 51^{\prime} 41,30^{\prime \prime}$ Oeste: $56^{\circ} 04^{\prime} 08,60^{\prime \prime}$ \\
\hline Pop. 2 & Alta Floresta- MT & Sul: 952'29,30" Oeste: $56^{\circ} 04^{\prime} 46,49^{\prime \prime}$ \\
\hline Pop. 3 & Alta Floresta - MT & Sul: 952'40,60" Oeste: $56^{\circ} 03^{\prime} 53,22^{\prime \prime}$ \\
\hline
\end{tabular}

A viabilidade polínica foi estimada através da utilização de dois corantes: a solução de 2,3,5-cloreto de trifeniltetrazólio (TTC) e lugol 2\%. Os botões coletados foram imediatamente usados para a preparação do experimento com o corante 2,3,5 cloreto trifeniltetrazólio (TTC) por agir em enzimas desidrogenases ativas. As anteras foram colocadas em placas de cultura celular com capacidade de $3 \mathrm{~mL}$ cada célula da placa em duas concentrações: $0,075 \%$ e $0,30 \%$, após, as anteras foram maceradas para a liberação dos grãos de pólen.

$\mathrm{O}$ material foi conduzido em ambiente de temperatura controlada de $23^{\circ} \mathrm{C}$ testados com presença e ausência de luz, e avaliados em diferentes períodos, sendo, 6:00, 12:00, 18:00 e 24:00 horas de exposição. Para o preparo das lâminas utilizou-se aproximadamente $0,5 \mathrm{~mL}$ de cada tratamento e cobriu-se a lâmina com 
uma lamínula. Para cada tratamento foram preparadas cinco lâminas e contabilizados 300 pólens. Sendo assim analisou-se 24.000 pólens para cada população, perfazendo um total de 72.000 pólens. Os grãos de pólen foram considerados viáveis quando apresentaram tonalidade em vermelho e inviáveis aqueles que não apresentaram coloração.

Para a preparação das lâminas com o corante lugol $2 \%$ as anteras foram depositadas nas lâminas e maceradas com cerca de $0,5 \mathrm{~mL}$ de corante. Foram preparadas 10 lâminas para cada população e contabilizados 300 grãos de pólen, totalizando 3.000 grãos contados, sendo armazenadas a $4^{\circ} \mathrm{C}$ e observados 24 horas após a preparação para que o corante reagisse com o amido dos grãos de pólen. Foram considerados viáveis os pólens que apresentaram tonalidades mais escuras e inviáveis aqueles que apresentaram tonalidades mais claras.

As lâminas dos grãos de pólen foram analisadas em objetiva de $40 \mathrm{X}$ em microscópio fotômico binocular (Leica ICC 50) acoplado a um computador e no software LAZ EZ V1. 7.0. Após a análise das lâminas, calculou-se a percentagem de pólens viáveis por meio da fórmula: №. de grãos corados/ №. de grãos contados * 100 e, posteriormente, submetidos à análise de variância e as médias comparadas pelo teste de Tukey ao nível de $5 \%$ de probabilidade através do programa $R$, versão 3.3.2 (R CORE TEAM, 2016), com o auxílio do pacote ExpDes, versão 1.1.2 (FERREIRA et al., 2013) e, para as variáveis significativas perante a análise de variância, foram geradas equações de regressão.

\section{RESULTADOS E DISCUSSÕES}

$\mathrm{Na}$ avaliação da viabilidade polínica por meio do corante TTC nas três populações (tabela 1) foi observada que a taxa de viabilidade é baixa segundo SOUZA et al., (2002). Na exposição de seis horas com concentração de 0,30\% sem presença de luz, a maior taxa de viabilidade polínica foi de 15,00\% (Pop.2). Com 12 e 18 horas de exposição as maiores taxas de grãos de pólens viáveis foram na população 3 na concentração de 0,30\% (36,26\% e 41,86\%) na ausência de luz. Na exposição de 24 horas no TTC $0,30 \%$ de concentração na ausência de luz da população 3 foi observado maior percentual de viabilidade com 18,80\%. Segundo LAUTON et al., (2016) estudando a espécie Plumeria obtiveram na concentração 0,075\% de TTC médias baixas de viabilidade polínica entre 0 a 2,40\%.

O teste de tetrazólio baseia-se na atividade das enzimas desidrogenadas, durante a glicólise e o ciclo de Krebs, ocorre a catalisação das atividades respiratórias nas mitocôndrias. O sal do tetrazólio (2,3,5-trifenil cloreto de tetrazólio ou TTC) ocorre redução nos tecidos vivos. Desta forma a reação de redução faz com que o composto incolor se transforme em um composto vermelho, estável e nãodifusível, conhecido como trifenilformazan, logo quando há atividades respiratórias nas mitocôndrias o TTC é reduzido, formando o trifenilformazan, corando as mesmas, indicando assim a viabilidade da célula e do tecido. Portanto as células que apresentam coloração indicam que há respiração celular indicando que esta é viável e as células inviáveis não reagem logo estas não se coram. 
TABELA 01: Valores da viabilidade dos grãos de pólen de Syzygium malaccense (L.) MERR. \& PERRY, pela coloração com TTC em concentrações de $0,075 \%$ e $0,30 \%$, em duas diferentes temperaturas e quatro tempos de exposição.

\begin{tabular}{|c|c|c|c|c|c|c|}
\hline \multirow{2}{*}{ Poulações } & \multirow{2}{*}{ Concentração } & \multirow{2}{*}{ Luz } & \multicolumn{4}{|c|}{ Tempos } \\
\hline & & & 6 & 12 & 18 & 24 \\
\hline \multirow{4}{*}{01} & $0,075 \%$ & Claro & $0,00 \mathrm{Bb}$ & $0,46 \mathrm{Bb}$ & $4,97 \mathrm{Bb}$ & $0,40 \mathrm{Bb}$ \\
\hline & $0,30 \%$ & Claro & $0,00 \mathrm{Bb}$ & $0,19 \mathrm{Bd}$ & $1,26 \mathrm{Bd}$ & $0,06 \mathrm{Bd}$ \\
\hline & $0,075 \%$ & Escuro & $0,00 \mathrm{Bb}$ & $0,26 \mathrm{Bb}$ & $0,33 \mathrm{Bb}$ & $0,06 \mathrm{Bb}$ \\
\hline & $0,30 \%$ & Escuro & $0,00 \mathrm{Bb}$ & $0,06 \mathrm{Bd}$ & $0,06 \mathrm{Bd}$ & $0,73 \mathrm{Bd}$ \\
\hline \multirow{4}{*}{02} & $0,075 \%$ & Claro & $1,13 \mathrm{Bb}$ & $1,13 \mathrm{Bb}$ & $1,13 \mathrm{Bb}$ & $0,39 \mathrm{Bb}$ \\
\hline & $0,30 \%$ & Claro & $10,46 \mathrm{Aa}$ & $10,49 \mathrm{Ab}$ & $16,73 \mathrm{Ac}$ & $18,53 \mathrm{Ab}$ \\
\hline & $0,075 \%$ & Escuro & $0,00 \mathrm{Bb}$ & $0,00 \mathrm{Bb}$ & $0,40 \mathrm{Bb}$ & $8,06 \mathrm{Ba}$ \\
\hline & $0,30 \%$ & Escuro & $15,00 \mathrm{Aa}$ & $15,76 \mathrm{Aa}$ & $31,06 \mathrm{Ab}$ & $26,53 \mathrm{Aa}$ \\
\hline \multirow{4}{*}{03} & $0,075 \%$ & Claro & $0,00 \mathrm{Bb}$ & $0,00 \mathrm{Bb}$ & $0,40 \mathrm{Bb}$ & $0,20 \mathrm{Bb}$ \\
\hline & $0,30 \%$ & Claro & $10,60 \mathrm{Aa}$ & $36,26 \mathrm{Aa}$ & $41,86 \mathrm{Aa}$ & $7,20 A c$ \\
\hline & $0,075 \%$ & Escuro & $0,20 \mathrm{Bb}$ & $3,33 \mathrm{Bb}$ & $1,20 \mathrm{Bb}$ & $0,46 \mathrm{Bb}$ \\
\hline & $0,30 \%$ & Escuro & $5,00 \mathrm{Bb}$ & $21,46 \mathrm{Ab}$ & $12,26 \mathrm{Ac}$ & $18,80 \mathrm{Ab}$ \\
\hline CV (\%) & & & 35,28 & & & \\
\hline
\end{tabular}

Médias seguidas pela mesma letra maiúscula nas linhas minúscula nas colunas não diferem pelo teste de scottknott a nível de $5 \%$ de significância.

Conforme HORNER \& PALMER (1995) o surgimento de grãos de pólen anormais, ou inviáveis, pode ser resultado de aberrações estruturais e numéricas, que por sua vez, decorrem de anormalidades que podem ocorrer durante a prémeiose, meiose e pós-meiose. NETO et al., (2006) afirmam que, para assegurar o sucesso nas hibridações controladas, é importante que o pólen a ser utilizado tenha boa viabilidade. Para JUNIOR et al., (2015) baixas taxas de viabilidade polínica foram observadas em Sisyrinchium commutatum (Iridaceae) em consequencia de irregularidades na meiose que podem estar associados a fatores abióticos diversos.

Por meio da coloração com lugol $2 \%$ (tabela 2) foi possível verificar a viabilidade polínica das três populações de Syzygium malaccense classificando-a como baixa para média de $9,46 \%$ a $57,46 \%$ de acordo com SOUZA et al., (2002). Desta forma os resultados da viabilidade polínica obtida com uso do TTC e com lugol $2 \%$ coincidem e confirmam os resultados obtidos. Certamente as taxas de viabilidade polínica estejam associadas principalmente aos fatores abióticos como baixa umidade do ar, que interfere na qualidade dos grãos de pólen.

$\mathrm{Na}$ figura 1, a população $2 \mathrm{com} 0,075 \%$ (Fig.A) e 0,30\% (Fig.B) de concentração de TTC tanto com a presença e ausência de luz, houve aumento linear na coloração na viabilidade polínica em relação ao tempo de exposição dos grãos de pólen. O maior tempo de exposição permitiu maior e melhor reação das enzimas 
desidrogenadas, durante a glicólise e o ciclo de Krebs, esteja ainda associado a organização da intine e exine dos grãos de pólen de Syzygium malaccense. Para a população $3 \mathrm{com} 18$ horas de exposição com presença de luz foi estimada maior taxa de viabilidade polínica (Fig.1 C) que esta associada ainda a maturidade destes grãos de pólen.
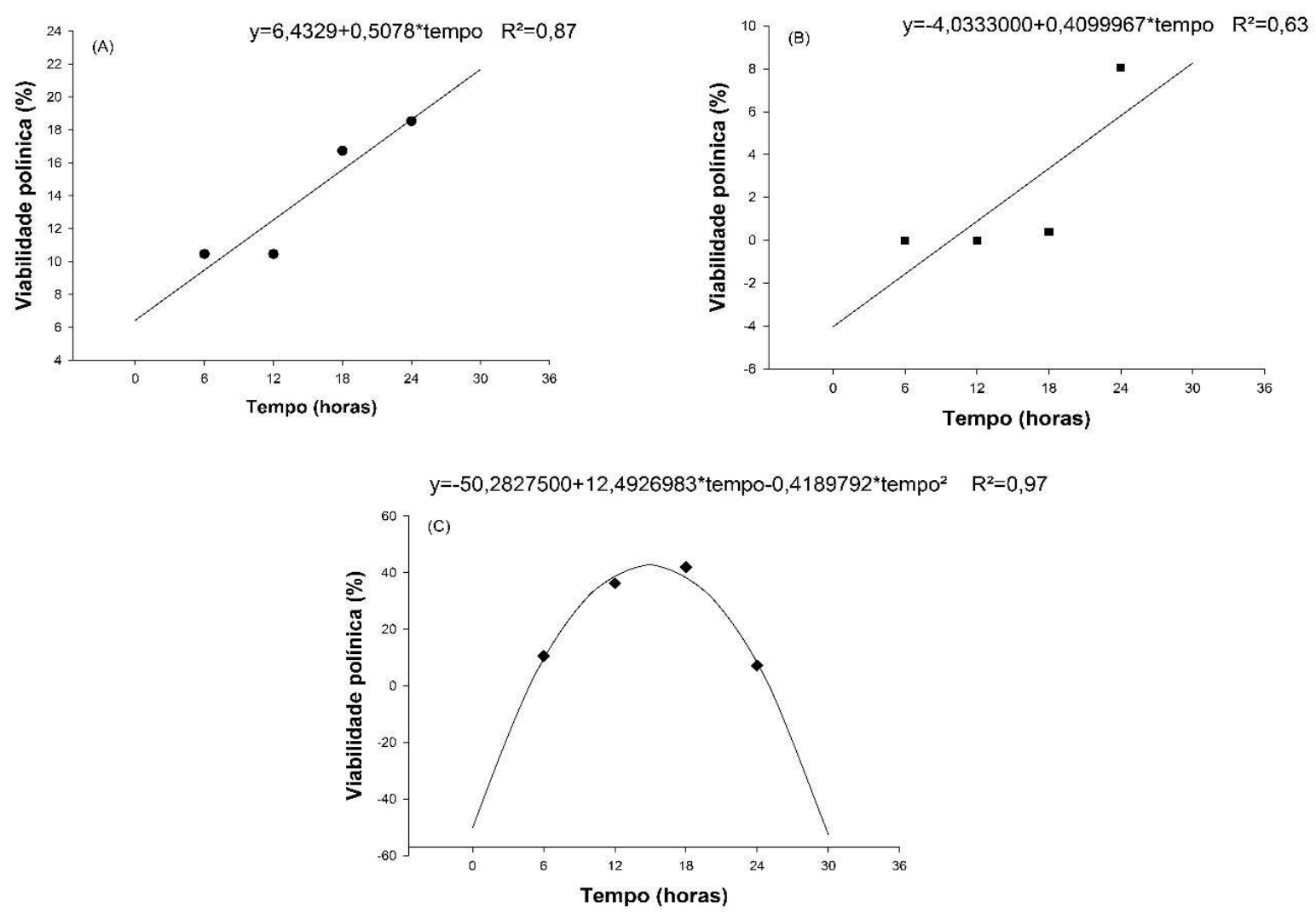

FIGURA 1. Percentual de viabilidade polinica de Syzygium malaccense (L.) MERR. \& PERRY com o uso de tetrazólio em função do tempo. (A) P2 0,30\% de TTC claro; (B) P2 0,075\% de TTC escuro e (C) P3 0,30\% de TTC claro.

$\mathrm{Na}$ tabela 2, ao utilizar o corante lugol $2 \%$ foi possível constatar que as populações 02 e 03 obtiveram médias de $48,36 \%$ e $57,46 \%$ de viabilidade polínica respectivamente não havendo diferença estatística entre si, sendo considerada como viabilidade média, a população 01 teve média $9,46 \%$ sendo considerada de baixa viabilidade diferindo estatisticamente das demais populações, conforme SOUZA et al. (2002). Essa baixa viabilidade está ligada a fatores abióticos que interferiram na formação dos gametas e irregularidades que ocorreram durante o processo de meiose, ou relacionada com os diferentes estágios de desenvolvimento dos botões florais.

A coloração com lugol baseia-se em uma reação química que acontece entre o iodo e a molécula de amido, dando aos grãos de pólen viáveis uma coloração marrom e aos inviáveis, devido à ausência de amido, uma coloração amarela clara, é indicado para espécies cujo material de reserva do grão de pólen é o amido 
(PAGLIARINI \& POZZOBON, 2004). Além disso, foi possível constatar que o lugol foi eficiente, podendo assim observar nitidamente os grãos de pólen viáveis e inviáveis a partir da coloração presente. Em estudos anteriores da espécie Delonix regia foram utilizados vários tipos de corante constando que o de maior eficiência foi o Lugol $1 \%$, pois foi o corante que melhor diferenciou os grãos de pólens viáveis de inviáveis, assim comparado a este estudo (NASCIMENTO et al., 2014).

Segundo NETO et al., (2006) em estudo com seis populações da espécie Solanum paniculatum $\mathrm{L}$. constatou-se uma população com viabilidade média de $67,5 \%$ comparada ao presente estudo da espécie Syzygium malaccense em que se observaram duas populações com viabilidade média de 48,36\% e 57,46\%.

TABELA 02: Médias percentuais de viabilidade do pólen entre as populações de Syzygium malaccense (L.) Merr. \& Perry, estimada pelo corante lugol $2 \%$.

\begin{tabular}{cc}
\hline Populações & Viabilidade polínica (\%) \\
\hline $\mathbf{0 1}$ & $9,46^{\mathrm{b}}$ \\
$\mathbf{0 2}$ & $48,36^{\mathrm{a}}$ \\
$\mathbf{0 3}$ & $57,46^{\mathrm{a}}$ \\
\hline $\mathbf{C V}(\%)$ & 28,55 \\
\hline
\end{tabular}

Médias seguidas pela mesma letra minúscula na coluna não diferem pelo teste de tukey a nível de $5 \%$ de significância.

Para explicar percentuais de viabilidade, o método utilizado para coloração (TTC) de pólen não se mostrou adequado. Esse resultado se explica devido as alterações fisiológicas que ocorrem no grão de pólen, um exemplo de decréscimo de viabilidade, foi a redução da respiração celular que ocorreu durante o tempo de exposição ao TTC. Segundo STANLEY \& LINSKENS (1974) são exemplos de tais alterações existentes, a alteração na conversão do açúcar, velocidade de respiração, ácidos orgânicos, na exina do pólen e no acúmulo de produtos metabólicos secundários. Observou-se que na ausência de luz algumas médias de viabilidade polínica foram maiores que na presença desta. Isso se explica pelo fato que a solução de tetrazólio é fotossensível.

\section{CONCLUSÃO}

$\mathrm{Na}$ estimativa dos grãos de polens do Syzygium malaccense, o corante lugol $2 \%$ apresentou maiores taxas de viabilidade em relação ao TTC, sendo que este corante tem afinidade por amido e óleos presentes na intine do pólen. Sendo o lugol $2 \%$ uma possibilidade confiável de uso para esta espécie. Pois com o corante TTC na concentração de $0,30 \% \mathrm{com} 18$ horas de exposição sem a presença de luz as taxas de viabilidade estão correlacionadas com os valores obtidos por meio do lugol $2 \%$. Assim, ambos corantes podem ser utilizados para estimar a viabilidade polínica da espécie em questão, dependendo das condições para a avaliação, bem como da possibilidade de análise no momento da coleta.

\section{REFERÊNCIAS}

BRAMBATTI, A.; BRAMMER, S. P.; WIETHÖLTER, P.; JUNIOR, A. N. Estabilidade genética em triticale estimada pela viabilidade polínica. Arquivos do Instituto biológico. v.83, 1-7, e0802014, 2016. DOI: 10.1590/1808-1657000802014 AGRARIAN ACADEMY, Centro Científico Conhecer - Goiânia, v.4, n.7; p.480 2017 
CAVALCANTE, P.B. Frutas comestíveis da Amazônia. 6.ed. Belém: CNPq/Museu Paraense Emílio Goeldi, 1996. 279p.

CEAGESP. Companhia de Entrepostos e Armazéns Gerais do Estado de São Paulo. Disponível em: <www.ceagesp.gov.br> Acesso em 12 de junho de 2017.

DONADIO, C. D.; NACHTGAL, J.C.; SACRAMENTO, C. K. Frutas exóticas. Jaboticabal: $\quad$ FUNEP, 1998. 279p. GARWOOD, N.C. Morphologyandecologyofseedlings,

FERREIRA, E.B.; CAVALCANTI, P.P.; NOGUEIRA, D.A. ExpDes: Experimental Designs package. $R$ package version 1.1.2. 2013. www.scielo.br/scielo.php?script=sci_nlinks\&ref=000141\&pid=S1413....Ing..

HORNER, H. T.; PALMER, R. G. Mechanisms of genetic male sterility. Crop Science, MADISON, v. 35, n. 6, p. 1527-1535, 1995. lib.dr.iastate.edu/cgi/viewcontent.cgi?article=1047\&context.

JÚNIOR, R.S.DE A.; URDAMPILLETA, J.; GIL, A. B. Qualidade polínica, anormalidades meióticas e poliploidia em Sisyrinchium commutatum (Iridaceae). Rodriguésia 66(3): 923-929. 2015. DOI: 10.1590/2175-7860201566320

KUHN. A. W. Viabilidade polínica, Genotoxicidade, Efeito antiproliferativoe Composto fenólicos de PeltodonLongipes Kunth ExBenth. (Lamiaceae). Dissertação de Mestrado. Universidade Federal de Santa Maria- UFSM. Santa Maria/RS., 2015, 58 p.

KUROSAWA, C. Jambo-Vermelho. Disponível em: <http:/redeglobo.globo.com/cgibin/globoruralmontar_texto_ult.pl? controle=2040> acesso em: 12 de junho 2017

LAUTON, D. S.; SANTOS, A. C.; KARSBURG, I. V.; DAMASIO, J. F.; Viabilidade polínica de jasmim manga com corante tetrazólio. Ciência \& Tecnologia: Fatec-JB, Jabuticabal, v. $8, \quad$ n.1, 2016. Número especial 2. www.citec.fatecjab.edu.br/index.php/files/article/download/1057/pdf

MARTINS, L. A. R; LAVIOLA, B. G.; PRAÇA-FONTES, M. M. Viabilidade polínica de Jatropha curcas L.: uma comparação metodológica. In: Congresso Brasileiro de Mamona, 5; Simpósio Internacional de Oleaginosas energéticas 2. Fórum Capixaba de Pinhão Manso, 1. 2012, Guarapari. Desafios e Oportunidades: Anais... Campina Grande: Embrapa Algodão, 2012.

MARTINS, V. de C. Estudo da Estabilidade das Antocianinas Majoritárias do fruto do Jambo Vermelho (Syzygium malaccense L. Merry \& Perry) In: Reunião anual da Sociedade Brasileira de Química, v. 37, 2014, Natal. Anais.

MELO, R. R. Características farmacobotânicas, químicas e biológicas de Syzygium malaccense (L.) Merr. Et L. M. Perry. Revista Brasileira de Farmácia, v. 90, p. 298- 
302, 2009. www.rbfarma.org.br/.../pag_298a302_caracteristicas_farmacobotanicas_220_904.pdf

NASCIMENTO, L. S.; BENEVENUTI, A. S.; LEITE, D. M.; SILVA, D. D., MOURA, E. A.; MIRANDA, D.; MELLO V.; DAHMER, N.; KARSBURG, I. V.; Estimativa da viabilidade polínica e índice meiótico de Delonix regia. Estudos, Goiânia, v. 41, especial, p. 83-88, nov. 2014. seer.ucg.br/index.php/estudos/article/viewFile/3854/2195

NETO, O. D. S.; KARSBURG, I. V.; YOSHITOME M. y.; Viabilidade e germinabilidade polínica de populações de jurubeba (Solanum paniculatum L.). Revista de Ciências Agro-Ambientais, Alta Floresta, v.4, n.1, p.67-74, 2006. www.unemat.br/revistas/rcaa/docs/vol4/8_artigo_v4.pdf

ORGANIZAÇÃO PAN-AMERICANA DA SAÚDE. Área de Desarrollo Sostenible y Salud Ambiental. Evaluación de los Efectos de la Contaminación del Aire em la Salud de América Latina y el Caribe.Washington: Organização Pan-Americana da Saúde; 2017.

PAGLIARINI, M.S.; POZZOBON, M. T.(2004) II Curso de citogenética aplicada a recursos genéticos vegetais. Embrapa Recursos Genéticos e Biotecnologia - DF. http://portal.unemat.br/media/files/AVALIACAO\%20DA\%20VIABILIDADE\%20POLINI CA\%20EM\%20GENOTIPOS\%20DE\%20Theobroma\%20cacao\%20L_\%20COM\%20 BASE\%20EM\%20TESTES\%20COLORIMETRICOS.pdf.

R CORE TEAM. R: A language and environment for statistical computing. $R$ Foundation for Statistical Computing, Vienna, Austria. URL https://www.R project.org/. 2016.STANLEY, R. G.; LINSKENS, H. F. Pollen - biology, biochemistry and management. New York: Springer-Verlag, 1974. 172p.

SOUZA, M.M., PEREIRA, T.N.S. \& MARTINS, E.R. 2002. Microsporogênese e microgametogênese associadas ao tamanho do botão floral e da antera e viabilidade polínica em maracujá-amarelo (Passiflora edulis Sims f. flavicarpaDegener). Ciência e Agrotecnologia. Lavras 26:1209-1217.

http://www.scielo.br/scielo.php?script=sci_arttext\&pid=S0100-84042008000200003 